\title{
In Vivo Diagnostic Accuracy of Digital Radiography in the Detection of Secondary Caries in Anterior Teeth
}

\author{
Talaeipour AR ${ }^{1}$, Nemati Anaraki S'2, Heidarkhan Tehrani S ${ }^{3}$, Golpasha $\mathbf{M}^{4}$ \\ 'Professor, Oral and Maxillofacial Radiology Dept, Craniomaxillofacial Research Center, Dental Branch of Tehran, Islamic Azad University, Teh- \\ ran, Iran. \\ ${ }^{2}$ Assistant Professor,Operative Dentistry Dept, Dental Material Research Center, Dental Branch of Tehran, Islamic Azad University, Tehran, Iran. \\ ${ }^{3}$ Post-Graduate Student, Oral and Maxillofacial Radiology Dept, Dental Branch of Tehran, Islamic Azad University, Tehran, Iran. \\ ${ }^{4}$ Dentist
}

\begin{tabular}{l}
\hline ARTICLE INFO \\
\hline Article Type \\
Orginal Article \\
\hline Article History \\
Received: Oct 2015 \\
Accepted: Dec 2016 \\
ePublished: March 2016
\end{tabular}

Keywords:

Dental Caries,

Digital Radiography,

Diagnosis

\begin{abstract}
Background and Aim: Secondary caries are a common challenge for dentists. Many researchers have evaluated the accuracy of digital radiographic systems in the detection of secondary caries and have reported controversial results. Therefore, the aim of this in vivo study was to determine the diagnostic accuracy of digital radiography in the detection of secondary caries in anterior teeth.

Methods and Materials: In this diagnostic in vivo study, 34 patients were selected from among the individuals who wished to replace their anterior teeth restorations. The restorations in need of replacement were class III or class IV composite resin restorations which were at least 5 years aged with either a crack in the restoration body or with more than $0.5 \mathrm{~mm}$ marginal maladaptation or marginal discoloration. Digital radiographs were obtained and were observed randomly by four oral and maxillofacial radiologists. Caries detection was classified using a 5-point Likert scale. Statistics were computed to assess Kappa coefficients.

Results: According to the data, observer reliability for PSP sensor was between 0.79 and 0.88 which is an indicator of the high accuracy of PSP sensor. The sensitivity, specificity, positive predictive value, negative predictive value and accuracy were 90 , $77,86,85$ and $86 \%$ respectively.

Conclusion: The results suggest that in vivo digital radiography with PSP sensors is sufficiently accurate in the detection of secondary caries.
\end{abstract}

Please cite this paper as:

Talaeipour AR, Nemati Anaraki S, Heidarkhan Tehrani S, Golpasha M. In Vivo Diagnostic Accuracy of Digital Radiography in the Detection of Secondary Caries in Anterior Teeth. J Res Dent Maxillofac Sci. 2016;1(2):36-40.

*Corresponding author:

Sanaz Heidarkhan Tehrani,

Email: sanazhtehrani@gmail.com 


\section{Introduction:}

Secondary caries are a common challenge for dentists. They develop adjacent to the preexisting restorations due to microleakage, inadequate extension of the restoration's margins or insufficient caries removal. (1) The development of secondary caries might lead to failure of the restoration. (2) GV Black has defined the secondary caries as the reversible recurrence of caries at the margins of a restoration. ${ }^{(3)} \mathrm{A}$ suitable caries diagnostic technique must allow the detection of the disease at its primary stages. ${ }^{(4)}$ Several methods have been used to investigate secondary caries including clinical examinations in a cleaned and dried setting under sufficient light, visual inspection, and tactile sense by use of a dental explorer and dental floss and of course radiography. ${ }^{(5)}$

Unfortunately, a comprehensive technique with high sensitivity and accuracy for the early detection of caries is not yet available. ${ }^{(4,6,7)} \mathrm{Re}$ cently, researches have compared the diagnostic accuracy of digital radiography systems in caries detection with that of conventional film system. (4) Several advantages have been reported for intraoral digital radiographic systems such as low radiation exposure, convenience of storage, elimination of the darkroom equipment and wet procedure and dynamicity. The mentioned method has been claimed to be patient friendly and less time-consuming. ${ }^{(8)}$ However, the accuracy of imaging-based diagnosis has remained controversial. ${ }^{(1,2,5)}$ Photostimulable Phosphor (PSP) sensors are currently being used for digital imaging. Limited information is at hand on the capability of PSP over other digital radiographic systems in the in vivo detection of secondary caries.$^{(9)}$ Since secondary caries determine the clinical lifetime of a restoration, an efficient radiographic method seems essential. ${ }^{(10,11)}$ Therefore, the aim of the current study was to assess the diagnostic accuracy of intraoral digital PSP sensor in the detection of secondary caries in anterior teeth compared to visual and tactile inspections.

\section{Methods and Materials:}

This double-blind in vivo experiment involved 34 patients that were selected from among the individuals who wished to replace their anterior teeth restorations. The restorations in need of replacement were class III or class IV composite resin restorations which were at least 5 years aged with either a crack in the restoration body or with more than $0.5 \mathrm{~mm}$ marginal maladaptation or marginal discoloration. The restorations were candidates for periapical radiography based on the FDA and ADA guidelines. ${ }^{(12,13)}$ Consequently, 80 dental radiographs were taken and included in this study. ${ }^{(13)}$

Obtaining the radiographs

Digital periapical radiographs were obtained using Digora Optime PSP sensors (Soredex, Helsinki, Finland) with $31 \times 41 \mathrm{~mm}$ active area and $64 \mu \mathrm{m}$ pixel size. The exposures were made by Minray radiographic device (Soredex, Helsinki, Finland) with exposure settings of $70 \mathrm{kVp}, 0.2$ seconds and $7 \mathrm{~mA}$. Distance was adjusted using a sensor holder (Kerr, Middleton, WI, USA). After each exposure, the sensor was scanned immediately with Digora scanner and the images were exported to computer and saved in Tagged Image File Format (TIFF).

Observation and assessment of radiographs

Four oral and maxillofacial radiologists randomly observed the true size radiographs in a semi-dark room on a 17-inch monitor (Dell, Inspiron N65, China, KS) with an effective resolution of 1366 $\times 768$ pixels. The observer-monitor distance was $20-30 \mathrm{~cm}$. No time limit was set for observation of the radiographs. Observers viewed all radiographs and recorded their opinions in a data sheet using a five-point Likert scale as follows:

1. Definite absence of secondary caries

2. Probable absence of secondary caries

3. Undetectable

4. Probable presence of secondary caries

5. Definite presence of secondary caries Study Protocol

In this study, the radiographs were obtained from 34 patients ( 80 periapical radiographs) with class III or class IV composite resin restorations. All patients underwent visual and tactile (by use of a dental explorer) clinical examination under sufficient light in accordance with the conditions set forth by the FDA and ADA. Para-clinical results 
were validated by Golden standard which is the direct visual observation and checking the consistency and strength of the cavity walls after removal of the restoration ${ }^{(14)}$. Therefore, composite resin restorations were removed by use of a high-speed handpiece (Bien Air, Swiss made) and a diamond bur (Piranha 008, SS WHITE, USA).

Statistical analysis

Diagnostic parameters including the sensitivity, specificity, positive predictive value (PPV) and negative predictive value (NPV) and accuracy were calculated and analyzed. Statistics were computed to assess Kappa coefficients.

\section{Results:}

According to the data, Intra-observer kappa coefficients were between 0.79 and 0.88 which is an indicator of the high accuracy of PSP sensors. Additionally, the sensitivity, specificity, PPV and NPV values of digital radiography in the detection of secondary caries have been calculated. The sensitivity, specificity and accuracy of digital radiography were $90 \%, 77 \%$ and $86 \%$, respectively. Moreover, the PPV and NPV value of digital radiography were $86 \%$ and $85 \%$, respectively.

\section{Discussion:}

To the best of our knowledge, limited information is available regarding the in vivo diagnostic accuracy of digital radiography in the detection of secondary caries. According to the results of the present study, the sensitivity, specificity, PPV and NPV and accuracy of intraoral digital radiography in the diagnosis of secondary caries adjacent to Class III and Class IV composite resin restorations were 90 , $77,86,85$ and $86 \%$, respectively.

The accuracy of digital radiography in the detection of secondary caries is rather controversial. Different radiographic systems such as cone beam computed tomography (CBCT), Charge-Coupled Device (CCD) and PSP show different accuracies in caries detection. ${ }^{(13)}$ A recent study on the diagnostic accuracy of two different resolutions of PSP digital intraoral receptors in the detection of secondary caries, has reported no significant differences in sensitivity, specificity, PPV, NPV and accuracy of the two resolutions ${ }^{(14)}$. Furthermore, another in vitro study has compared the accuracy of digital and conventional radiography in the detection of recurrent caries and has concluded that there is no significant difference between digital and conventional radiography in the detection of recurrent caries. (15) Recently, researchers have evaluated the diagnostic accuracy of the reverse contrast mode in intraoral digital radiography in the detection of proximal dentinal caries. ${ }^{(16)}$ Based on their observations, the sensitivity, specificity, positive predictive value, negative predictive value, and accuracy of intraoral digital radiography were $72.5,90,87.2,76.5$ and $80.9 \%$, respectively. Whereas these values for the reverse contrast mode were $63.1,89.4,87.1,73.5$ and $78.8 \%$ respectively, which were lower compared to that of the original digital radiograph. Higher sensitivity value and similar accuracy value were ensued by the technique used in the current study. An In vitro study on the diagnostic ability of visual inspection, film, CCD sensor, PSP sensor and CBCT in the detection of proximal caries in posterior teeth compared to the gold standard, revealed that the intra-observer kappa coefficients calculated for each observer and for each radiographic modality ranged from 0.739 to 0.928 . ${ }^{(17)}$ Furthermore, inter-observer agreement for all modalities equaled 0.63 to 0.81 . There was no significant difference between the diagnostic ability of various techniques in the detection of proximal caries in posterior teeth. Our results concur with the results of the mentioned report. Another report concluded that PSP sensor was similar to Insight film in the detection of proximal enamel caries. The sensitivity, specificity and accuracy of Digora white plate were 14, 93 and 59\% while for Digora blue plate these values equaled 15, 94, 61\%, respectively. ${ }^{(18)}$ The sensitivity and accuracy of the technique used in our study were higher compared to the study by Pontual et al. ${ }^{(18)}$ Recently, the diagnostic ability of PSP plates in the detection of proximal caries has been studied with direct measurement by stereomicroscope. The researchers claimed that PSP plates are efficient in the diagnosis of proximal caries. ${ }^{(9)}$ The difference in observations might be attributed to different factors such as difficulty of detection of initial caries in enamel surface and/or the use of 
older version of sensors. Perhaps, newer digital systems have higher sensitivity than the older systems. ${ }^{(19,20)}$

An in vitro research that evaluated direct digital radiography in the diagnosis of occlusal caries in primary teeth, reported that direct digital radiographic technique is more effective than conventional radiographic examination and visual inspection. ${ }^{(21)}$ Abesi et al. stated that there was no difference between the accuracy of conventional and digital images by evaluating the radiographs of 72 non-cavitated posterior teeth ${ }^{(4)}$. Based on their observations, the specificity of all systems was $100 \%$ whereas the sensitivity of conventional film, PSP and CCD was 55, 55 and $45 \%$, respectively. Based on a research by Kayipmaz et al., no significant differences were detected among conventional intraoral radiography, PSP plate and CBCT in the detection of proximal caries. ${ }^{(22)}$ Presumably, the differences between our results and the results of the above investigation can be attributed to the evaluation of intact proximal surfaces in the mentioned study. However, further research is needed to determine the accuracy of the results. Li et al. evaluated the diagnostic accuracy of digital radiographs in proximal caries detection in 39 non-cavitated teeth which were involved in a neoplasm of the jaw ${ }^{(13)}$. The radiographs were taken prior to and after the surgery. Based on their observations, there was no significant difference between in vivo and in vitro digital radiographs. Perhaps, in the detection of proximal caries by digital radiography, in vitro condition may be similar to the real clinical situations. ${ }^{(23)}$

The accuracy of imaging-based diagnosis remains controversial since misinterpretations may occur due to the presence of radiolucent base and liner dental materials that mimic the appearance of secondary caries. ${ }^{(1,2,5)}$ It should be noted that the current study has been the first to evaluate the accuracy of digital radiography directly in the oral cavity. Therefore, confounding factors might manipulate the accuracy of the results. Presumably, the results of the present study are more accurate and realistic compared with the results of similar in vitro studies.

\section{Conclusions:}

It seems that in vivo digital radiography with PSP sensors is sufficiently accurate in the detection of secondary caries.

\section{Acknowledgement}

We are thankful to those who helped us in this article including research center of Islamic Azad University, Dental Branch of Tehran.

\section{References:}

1. Qu X, Li G, Zhang Z, Ma X. Detection accuracy of in vitro approximal caries by cone beam computed tomography images. Eur J Radiol 2011; 79(2): e24-27. 2. Lachowski KM, Botta SB, Lascala CA, Matos AB, Sobral MA. Study of the radio-opacity of base and liner dental materials using a digital radiography system. Dentomaxillofac Radiol 2013; 42(2):20120153. 3. Mjör IA, Toffenetti F. Secondary caries: A literature review with case reports . Quintessence Int 2000; 31(3):165-79.

4. Abesi F, Mirshekar A, Moudi E, Seyedmajidi M, Haghanifar S , Haghighat N, et al. Diagnostic Accuracy of Digital and Conventional Radiography in the Detection of Non-Cavitated Approximal Dental Caries.Iran J Radiol 2012; 9(1): 17-21.

5. Senel B, Kamburoglu K, Uçok O, Yüksel SP, Ozen T, Avsever H. Diagnostic accuracy of different imaging modalities in detection of proximal caries. Dentomaxillofac Radiol 2010; 39(8):501-11.

6. Haak R, Wicht MJ, Noack MJ. Conventional, digital and contrast-enhanced bite-wing radiographs in the decision to restore proximal carious lesions. Caries Res 2001;35(3):193-9.

7. Van der Stelt PF. Filmless imaging: the uses of digital radiography in dental practice. J Am Dent Assoc 2005; 136(10): 1379-87.

8. Wenzel A, Hirsch E, Christensen J, Matzen LH, Scaf G, Frydenberg M. Detection of cavitated approximal surfaces using cone beam CT and intraoral receptors. Dentomaxillofac Radiol 2013;42(1):39458105.

9. Vivek V, Thomas S, Nair BJ, Vineet AD, Thomas J, Ranimol P, Vijayan AK. Comparison of diagnostic ability of storage phosphor plate in detecting proximal caries with direct measurement by stereomicroscope: a pilot study. Clin Pract 2015;28;5(3):763-70.

10. Moreira PL, Messora MR, Pereira SM, Almeida $\mathrm{SM}$, Cruz AD. Diagnosis of secondary caries in esthetic restorations: influence of the incidence vertical angle of the X-ray beam. Braz Dent J 2011; 22(2): 129-33.

11. Gimenez T, Piovesan C, Braga MM, Raggio DP, Deery C,Ricketts DN,et al. Visual Inspection for Car- 
ies Detection: A Systematic Review and Meta-analysis. J Dent Res. 2015; 94(7):895-904.

12.American Dental Association Council on Scientific Affairs. The use of dental radiographs: update and recommendations. The Journal of the American Dental Association 2006;137(9):1304-12.

13. Li G, Qu XM, Chen Y, Zhang ZY, Ma XC . Diagnostic accuracy of proximal caries by digital radiographs: an in vivo and in vitro comparative study. Oral Surg Oral Med Oral Pathol Oral Radiol Endod 2010;109 (3):463-7.

14. Mehralizade S, Nemati Anaraki S, Sakhdari S, Miraba S, Amiri Siavashani M, Bayat S. Comparing the diagnostic accuracy of two different resolution for PSP digital intraoral receptors in detection of secondary caries (In Vitro). JRDMS 2015; 1(1): 40-5.

15. Anbiaee N, Mohassel AR, Imanimoghaddam M, Moazzami SM. A comparision of the accuracy of digital and conventional radiography in the diagnose of recurrent caries.J Contemp Dent Pract 2010;11(6):2532.

16. Miri S, Mehralizadeh S, Sadri D, Motamedi MR, Soltani P. The efficacy of the reverse contrast mode in digital radiography for the detection of proximal dentinal caries. Imaging Sci Dent 2015; 45(3): 141-5. 17.Pontual AA, de Melo DP, de Almeida SM, Bóscolo FN, Haiter Neto F. Comparison of digital systems and conventional dental film for the detection of approximal enamel caries. Dentomaxillofac Radiol 2010; 39(7):431-6.

18.Haiter-Neto F, dos Anjos Pontual A, Frydenberg $\mathrm{M}$, Wenzel A.A comparison of older and newer versions of intraoral digital radiography systems: diagnosing noncavitatedproximal carious lesions. J Am Dent Assoc 2007; 138(10):1353-9.

19. Esiefarienrhe BM, Amuche AA. Computer-based radiological laboratory diagnosis system. IJSES 2016;2(1):12-21.

20. Dias da Silva PR, Martins Marques M, Steagall W Jr, Medeiros Mendes F, Lascala CA. Accuracy of direct digital radiography for detecting occlusal caries in primary teeth compared with conventionalradiography and visual inspection: an in vitro study. Dentomaxillofac Radiol 2010; 39(6):362-7.

21. Kayipmaz S, Sezgin ÖS,Saricaoğlu ST,Çan G.An in vitro comparison of diagnostic abilities of conventional radiography, storage phosphor, and cone beam computed tomography to determine occlusal and approximal caries.Eur J Radiol 2011; 80(2):478-82.

22. Matteson SR, Phillips C, Kantor ML, Leindecker $\mathrm{T}$. The effect of lesion size, restorative material, and film speed on the detection of recurrent caries . Oral surg Oral Med Oral Pathol 1989; 68(2):232-7.

23. Espelid I, Tveit AB, Erickson RL, , Glasspoole EA. Radioopacity of restorations and detection of sec- ondary caries. Dent Mat 1991; 7(2):114-7. 